\title{
Enseñanza de la sistematización de experiencias en el componente investigativo de la formación de profesionales de Educación Física, Universidad de Antioquia, Colombia
}

José Fernando Tabares

\section{RESUMEN}

El objetivo del trabajo fue la recuperación del seminario de investigación VII, ofrecido a los estudiantes del programa de educación física de la Universidad de Antioquia en Medellín-Colombia, en el segundo semestre académico de 2016. Para ello se adelantó una sistematización de experiencias, a partir de la información acopiada mediante la reconstrucción de la ruta con líneas del tiempo, recolección y organización de los registros, evaluación del proceso e interpretación crítica. Esto posibilitó dar cuenta de sus elementos constitutivos. En este sentido, se identificó esta forma de pesquisa como posibilidad de investigación formativa para la mayoría de los futuros profesionales que no se desempeñarán como investigadores, sino en los diferentes ámbitos de actuación; también permitió ponernos de frente con el objeto y las formas de la producción de conocimiento más pertinentes. Por último, el proceso contribuyó a identificar aspectos centrales que aportan al robustecimiento del Seminario.

Palabras clave: Formación profesional. Investigación. Educación física.

1 Doctor en ocio y potencial humano. Profesor asociado del Instituto Universitario de Educación Física de la Universidad de Antioquia. Medellín/Antioquia, Colombia. E-mail: josef.tabares@udea.edu.co 
Teaching of the systematization of experiences in the research component of the training of professionals of Physical Education, University of Antioquia, Colombia

\begin{abstract}
The objective of the study was the recovery of the research seminar VII, offered to the students of physical education from the University of Antioquia in Medellin, Colombia, in the second semester of 2016. This was ahead of a systematization of experiences, based on the information gathered through the reconstruction of the route with lines of time, collection and organization of records, evaluation of the process and critical interpretation. This made it possible to give an account of their constituent elements. In this sense, was identified this form of research such as possibility of formative research for the majority of future professionals that is will not play as investigators, but in the different fields of action; It also allowed to put us in front with the object and the most relevant forms of knowledge production. Finally, the process contributed to identifying central aspects that contribute to the strengthening of the seminar.
\end{abstract}

Keywords: Vocational training. Research. Physical education.

Ensino da sistematização de experiências no componente investigativo da formação de profissionais da Educação Física, Universidade de Antioquia, Colômbia

\title{
RESUMO
}

O objetivo deste trabalho foi a recuperação do seminário de pesquisa VII, oferecido aos estudantes do programa de educação física da Universidade de Antioquia, em Medellín-Colômbia, no segundo semestre acadêmico de 2016. Para isso, foi sistematizada a experiência, a partir das informações coletadas através da reconstrução da rota com linhas de tempo, coleta e organização dos registros, avaliação de processos e interpretação crítica. Isso tornou possível explicar seus elementos constituintes. Nesse sentido, essa forma de pesquisa foi identificada como uma possibilidade de pesquisa formativa para a maioria dos futuros profissionais que não realizarão como pesquisadores, mas nos diferentes campos de ação; também nos permitiu enfrentar o objeto e as formas mais relevantes de produção de conhecimento. Finalmente, o processo ajudou a identificar os aspectos centrais que contribuem para o fortalecimento do Seminário.

Palavras-chave: Formação profissional. Pesquisa. Educação física. 


\section{INTRODUCCIÓN}

La Universidad de Antioquia se inscribe en la perspectiva de las universidades investigadoras. Es decir, aunque promueve la docencia, la investigación y la extensión, su acento es hacia la producción de conocimiento. Enfoque con un sustancial énfasis en las ciencias básicas y sus lógicas propias. En este contexto, con estrategias como los semilleros adscritos a los grupos, se busca formar investigadores que luego puedan entrar a apoyar sus procesos investigativos y el relevo generacional. No obstante, la mayoría de los futuros profesionales se desempeñarán en campo, promoviendo y acompañando prácticas en diferentes contextos. Por lo que deberían tener unos conocimientos suficientes para poder reflexionar su práctica cotidiana y enriquecerla permanentemente.

La investigación en la formación profesional del programa de educación física de la Universidad de Antioquia, ha tenido una presencia preponderante de los abordajes positivistas, muy de la mano del predominio de tendencias salubristas y deportivistas. Esta situación se hace evidente en el mayoritario porcentaje de trabajos de grado. De acuerdo con Tabares, Molina y Cuervo $(2014$, p. 6$)$ de 167 informes presentados hasta el primer semestre de 2014, solo cinco, que equivalen al 3\% del total, corresponden a la sistematización de experiencias En este mismo sentido, en la indagación sobre la producción de conocimiento en los procesos formativos en el campo, en América Latina, se identificó para Colombia, un mayor registro de enfoques predominantemente instrumentales en las mallas curriculares de los programas de educación física.

En el currículo del programa, se registran ocho niveles de investigación. El seminario VII corresponde a la sistematización de experiencias y se ofrece en el séptimo semestre, es decir, cuando los y las estudiantes han pasado, para ese momento, por seis seminarios. Este aspecto es muy significativo, porque da cuenta de un proceso de formación investigativa que supone un sustancial acercamiento a sus diferentes perspectivas en el campo y que incide de manera considerable en sus ubicaciones epistemológicas, producto de las tendencias con mayor presencia.

En este contexto, el desarrollo del Seminario VII del segundo semestre de 2016, se dio fundamentalmente partiendo de la pregunta por la pertinencia de la educación física, el deporte, la recreación y la actividad física, en relación con el nuevo y esperanzador escenario del fin del conflicto y la construcción de la paz. O sea, el cuestionamiento por el papel que podría jugar en este marco. Esto puso en escena un primer elemento muy importante, la inquietud por el objeto del campo, es decir, por lo epistemológico. ¿Cuál es? ¿Se define a partir de una sola perspectiva o es diverso? ¿Cómo entonces abordarlo (s)? En últimas, qué tipo de campo es el más pertinente para pensar desde una sociedad en el posconflicto.

Y en esta misma línea ¿Qué papel juega la sistematización de experiencia como forma de la producción del conocimiento del campo?

Esta investigación se propuso la recuperación del proceso adelantado en el semestre II de 2016. Se buscó fortalecer el seminario mediante la reconstrucción crítica de la experiencia vivida. Como eje de este ejercicio, se definieron los procesos didácticos 
en la formación investigativa de profesionales en educación física, recreación, deporte y actividad física. Con estos elementos se hizo la sistematización de experiencias.

\section{Materiales y Métodos}

El estudio corresponde a una pesquisa crítica, en el sentido de una perspectiva cuyo objeto son las prácticas, que reivindica lo situado (local) y reconoce a las personas como sujetos de la investigación y que se propone contribuir mediante iniciativas colectivas, a la transformación de situaciones problemáticas de las comunidades. En consecuencia, emerge como forma de la producción del conocimiento que problematiza la situación de lo que Boaventura (2006) Ilama sociología de las ausencias. Es decir, la invisibilización de algunas formas que no se corresponden con las perspectivas hegemónicas.

La sistematización de experiencias florece en América Latina en la década de los 60', junto con otras iniciativas como la teología de la liberación, la economía del desarrollo, la educación popular y la IAP, en respuesta a las imposiciones propias del periodo de la doctrina del desarrollo en la región. Aunque es cierto que surge en las comunidades, luego encuentra apoyo en diferentes movimientos sociales y profesores universitarios, para el caso de Colombia con la propuesta de Orlando Fals Borda, que permiten la reconstrucción, ordenamiento e interpretación crítica de procesos innovadores, que después podrían ser compartidas con otros grupos y colectivos.

Aunque en el ámbito educativo esta forma de la producción de conocimiento creció de la mano de la educación popular, luego empezó a trabajarse en el ámbito educativo. Para el caso específico de la Educación Superior, su presencia no es muy significativa.

\section{La ruta de la sistematización de experiencias}

En referencia a la metódica de esta forma de investigación Jara (2012) propone una ruta que tiene en cuenta los siguientes pasos: un punto de partida que corresponde al hecho de haber participado en la experiencia y tener registros de ella. Un segundo momento es el de la definición de la práctica, el objetivo y el eje (aspecto central desde el que se recupera). El siguiente paso es la recuperación del proceso vivido, a través de la reconstrucción de su historia y del ordenamiento y clasificación de la información. Por último, está la reflexión de fondo, mediante una interpretación crítica de los principales asuntos y aprendizajes que emergen de él.

Por último está el momento de llegada, en el que se hacen recomendaciones, aprendizajes y propuestas y se genera alguna propuesta de comunicación (video, cartilla, metodología, artículo, guía, etc.). Para ello se acude a estrategias metodológicas tales como análisis documental, entrevistas, talleres, grupos focales, etc. 


\section{La sistematización de experiencias en el programa de educación física de la Universidad de Antioquia}

En el marco de las universidades investigadoras, cuyo eje central es la producción de conocimiento: artículos, libros, patentes y sobre todo de modelos de las llamadas ciencias duras, la pregunta es por la investigación formativa o la formación para la investigación. Es decir, formar solo investigadores o articular la pesquisa en los procesos de práctica profesional, de tal manera que esta contribuya a la reflexión permanente respecto a las formas del actuar.

Investigación construida en los espacios de la periferia, el margen o la exterioridad que representa en la actualidad una significativa opción de generación de conocimiento en territorios diversos como los nuestros. Este tipo de indagación ha tomado varias vertientes tales como la académica, propia de los procesos desarrollados en la universidad, con tendencia a trabajar desde modos con mayor cercanía a los formalismos del aparato burocrático que lo orienta. La de las agencias de cooperación nacionales e internacionales y más recientemente las entidades gubernamentales, predominantemente orientada hacia la evaluación de programas adelantados en algunos de los sectores propios de su objeto. Una última línea, es la que concibe la sistematización como forma crítica con una propuesta metodológica que se separa de las formas utilizadas en la universidad (al menos en su versión occidentalizada), para proponer una ruta que parte de la experiencia misma para recorrer el camino de su reconstrucción a partir de los actores sociales.

El seminario de sistematización se inscribe en la tercera tendencia. Por ello se centra en la ruta planteada por perspectivas ubicadas en procesos sociales y de educación popular. Para este caso específico se propone a manera de punto de partida la propuesta de Jara (1994), con algunos aportes de otros autores para ampliarla y fortalecerla. Más allá de cuestionar la preeminencia de la praxis como rastro por excelencia de nuestro quehacer profesional, se busca problematizar los binarios que soportan las formas normales de la investigación. Valga decir, teoría-práctica, local-global, sujeto-objeto, entre otros. Parto pues de las prácticas y de su potencial epistemológico (MEJÍA, 2013). O sea, busco trascender la discusión entre las prácticas y las teorías, buscando fortalecer la construcción del conocimiento desde estas primeras, a partir del reconocimiento de su complejidad.

\section{Las prácticas como objeto de estudio en el campo de la Educación Física}

En la Universidad de Antioquia, la influencia del Convenio Colombo-alemán firmando en 1974, con su óptica de ciencias del deporte, influyó de acuerdo con Pinillos (2011) en los procesos de formación, al configurar una noción que transformó la Educación Física, modificando sus fines, contenidos, medios y campos de acción, generando una oposición de discursos. O sea, el posicionamiento de un referente, con un fuerte acento en fisiología y anatomía que contribuiría al logro de un estatus científico para el área. Esto generó unas condiciones de posibilidad favorables a las tendencias que se 
inscriben en ella y de desventaja para las que no. Es decir, que este campo de estudios tuvo y sigue teniendo un énfasis instrumental en detrimento de otras miradas que acentúan la importancia de lo social.

Ante situaciones como el proceso de paz en nuestro país y un posible marco de posconflicto, la pregunta sobre la pertinencia del campo problematiza su actual situación. Sobre la poca presencia de modalidades como la sistematización de experiencias en los procesos de formación en investigación, la pregunta es por el escenario más adecuado, el que posibilite de mejor manera la integración de los diferentes factores de un contexto en el que apostamos por la reconstrucción de la dignidad, la equidad y el respeto de la diversidad en esta nación tan heterogénea.

Acá la labor de ampliar el espectro de formas de la producción de conocimiento más amplias e integradoras como la IAP y la sistematización de experiencias, debe ser una tarea de quienes consideramos que el campo de estudios requiere de miradas múltiples con variados acercamientos. Adelantaremos una reflexión sobre las condiciones de posibilidad de esta forma de investigación, buscando identificar sus principales aportes y dificultades en el entendido de que las prácticas representan un escenario privilegiado en nuestra profesión y que por tanto deberíamos prestarles una mayor atención.

La identidad de la Educación Física es un eje sobre el que pueden estructurarse y articularse las distintas partes que componen el campo; el problema se nos hace ineludible. Por un lado, se presenta de múltiples maneras en las prácticas de docencia y de investigación, oculto o develado en la heterogeneidad de las doctrinas para orientarla, en la diversidad de las tareas que nos ocupan en escuelas, clubes, gimnasios, hospitales, centros vacacionales; en la ambigüedad de las imágenes y atributos que los otros nos devuelven y solicitan; en las fisuras del ámbito laboral por las que se entran médicos, entrenadores, exdeportistas, por mencionar algunos. Por el otro, la pretensión de afirmar la existencia de un objeto científico que le corresponde solo a ella (CRISORIO, 2007, p. 1).

En la misma línea de Crisorio (2007), parto de la decisión de intentar mostrar el campo a partir de lo que sucede en contexto, es decir, desde la multiplicidad de prácticas que ejercen los profesionales en los diversos escenarios de actuación. Entre ellos programas comunitarios de promoción de la salud y la prevención de la enfermedad, escuelas de formación deportiva, gimnasios y centros de acondicionamiento físico, educación, actividades de aventura, primera infancia, entrenamiento personalizado, diversas manifestaciones recreativas, alto rendimiento, población discapacitada, por mencionar algunas. Prácticas que en últimas portan sus propias particularidades, imposibles de agrupar en un solo objeto y menos con unas formas exclusivas de la producción del conocimiento.

Educación física, ciencias del deporte, cultura física, motricidad, expresiones motrices, entrenamiento deportivo, educación corporal, praxiología, etc., son algunas nociones para representar la totalidad del campo. En la actualidad, puede considerarse la existencia de una tensión entre perspectivas, que pugnan por un espacio en dicho escenario. En esta situación, los profesionales se desempeñan en múltiples entornos, en donde deben desplegar un cúmulo de conocimientos a través de rutas metodológicas, acordes con la especificidad de los contextos y grupos humanos. Esto evidencia la necesidad 
de incluir formas de investigación orientadas sobre todo a la identificación de las mejoras desde rastros centrados en indicadores que muestren sí, la mejora de las cualidades y las capacidades físicas, pero también el horizonte hacia asuntos de tipo social, cultural y político. Es decir, centrar la mirada en las prácticas, entendidas como dispositivos que contienen en ellos mismos maneras de comprender la vida, la sociedad, el ser humano y claro, el área de estudios.

Las prácticas son portadoras de las formas como en lo local se configuran y reconfiguran los contextos. Desde esta perspectiva nuestro campo no es homogéneo ni las modalidades del conocimiento iguales. Por el contrario, la heterogeneidad de nuestros contextos reclama multiplicidad de miradas y abordajes. Es decir, la sistematización de experiencias, en ella misma, representa la posibilidad de una óptica de lo múltiple. Por ello contiene un enorme potencial para dar cuenta de alternativas subterráneas que la mirada de los modos de investigación predominantes no alcanzan a ver, porque se centran en lo verificable y cuantificable y los procesos de participación, de empoderamiento, de innovación, requieren de procedimientos cuidadosos de análisis de las prácticas, para encontrar en ellas y a partir de ellas, aspectos que pueden contribuir a la cualificación y fortalecimiento de las iniciativas adelantadas en múltiples lugares de nuestra ciudad.

De alguna manera es reivindicar el valor de la dimensión social de nuestro campo, tan invisible en ocasiones, pero tan presente en cada uno de los lugares en los que trabajamos con grupos de personas, desde la primera infancia hasta la vejez. En palabras utilizadas cotidianamente en los espacios de clase y en otros de la dependencia, es la necesidad de tener en cuenta el contexto. Ese intrincado asunto que impacta en los procesos que se adelantan en campo, donde muchas veces pareciera ser que los conocimientos adquiridos en la universidad no fueran los más pertinentes.

\section{Desarrollo del Seminario VII Sistematización}

En el año 2013, empiezo con el seminario de investigación VII. Como antecedente de este proceso, mi doctorado en ocio y potencial humano, cuya tesis trató el tema de la producción de conocimiento en este campo en Colombia, que mostró una presencia mayoritaria de enfoques empírico-analíticos y el bajísimo registro de perspectivas alternativas. Esta situación despertó mí interés en otras formas de pesquisa como la IAP y la sistematización de experiencias.

En ese primer seminario, retomo el trabajo de Jara (1994) para el diseño de la propuesta, pues la orientación que hasta ese momento se le había dado, correspondía a un tipo de autobiografía que hacía cada alumno. A partir de este año, inicio un proceso de posicionamiento de esta forma de pesquisa como ruta hacia la producción de conocimiento pertinente para el campo, tan orientado a tipos de indagación más cercanos a la óptica instrumental, correspondiente a tendencias biologicistas y del entrenamiento deportivo. En 2014, resultado de los procesos vividos y reconociendo la falta de referencias bibliográficas sobre esta manera de investigación en el área, diseñé con un profesor y un estudiante la 
Guía para el seminario, con intentos de articulación con la educación física, la recreación y el deporte, desde una perspectiva social.

En el 2016 orienté los seminarios, pero fue el del segundo semestre, el que representó, por el contexto, una dinámica que hizo visibles aspectos sobre los que ya venía cavilando.

Hasta el momento, resultado de la experiencia de los seminarios anteriores, dediqué la primera parte a tratar el tema de los modos de pesquisa en el campo y la predominancia de investigaciones de las ciencias médicas. El hecho de que en ese momento participara de una investigación sobre la producción de conocimiento en la formación profesional en educación física, recreación, deporte y actividad física con profesores de otros países de la región, me llevó a acercarme a la forma como se define el objeto y las formas del conocimiento.

En la marcha, identifiqué la necesidad de un tratamiento más cuidadoso del objeto del campo, para lo cual destiné tres encuentros, en los que a través de lecturas individuales y colectivas, discusiones y socializaciones, los estudiantes se acercaron a otras miradas que permitieron problematizar los lugares comunes en los que se ubicaban. ¡Un conocimiento situado, comprometido, participativo, desde la práctica...! Exclamaban. Este descentramiento, puede ser uno de los elementos a fortalecer en el seminario. Aunque algunos alumnos manifiestan su preferencia por las formas de indagación tradicionales, es primordial que reconozcan que el objeto se materializa en tensiones entre diferentes perspectivas.

Dediqué un segundo momento a la ruta de la sistematización de la experiencia. Acercar a los estudiantes a la metodología. Es estrategia del seminario, que se recupere una práctica en la que se haya participado. En esta dirección es conveniente mencionar que los alumnos pasan por ocho niveles de práctica. En muchos casos, son las que se sistematizan. En este proceso, identifiqué alguna dificultad, sobre todo en el tema del eje de la sistematización que debe ser trabajado con más cuidado, porque de ello depende la claridad para el resto de la ruta.

Paralelamente desarrollamos encuentros para el seguimiento del proceso y asesorías de acuerdo con las necesidades de cada proyecto. Esta estrategia que en los primeros seminarios se hacía individual o con grupos de estudiantes, empezó a hacerse con todo el grupo, al identificar que escuchando los procesos y dificultades de otros, se encontraban con respuestas y orientaciones para sus propios proyectos. Terminamos en lo que considero una propuesta muy cercana a una sistematización de experiencias.

El seminario de sistematización, correspondiente al segundo semestre de 2016, se desarrolló en el aula 45-208 de la sede Ciudadela Robledo de la Universidad de Antioquia en Medellín. A diferencia de los seminarios anteriores, en este, todos los estudiantes fueron hombres. Como está estipulado en el currículo, el primer encuentro se dedica a la presentación del programa y a la concertación de los aspectos básicos para su funcionamiento. También lo dedico al reconocimiento de las trayectorias de los alumnos y a sus intereses específicos, en referencia al ámbito de actuación de su preferencia. Además, realizo una conversación sobre el tema de la producción de conocimiento en el campo 
de la educación física, el deporte, la recreación y la actividad física, intentado ampliar la perspectiva frente a la temática.

En el primer momento, que corresponde a tres encuentros, como aprendizaje de los anteriores seminarios, se aborda un trabajo que busca que los estudiantes se cuestionen sobre la pertinencia de la sistematización de experiencias como forma de pesquisa del área. Esta acción encuentra un apoyo primordial en el contexto del plebiscito para la paz que se adelantaba en esos momentos. En el seminario, la pregunta por la oportunidad y contribución del campo a la construcción de la paz los cuestiona. La situación de que no es a partir de lo que creen o de lo que han leído o les han dicho en clase, sino desde un hecho concreto como el que se señala, interroga a los alumnos frente a las formas de indagación, intentando trascender algunas tendencias predominantes en la formación. Acá se trabaja con los documentos de Vaz (1999) y Crisorio (2007), para el tema del objeto, con el texto de Torres (2014), para la investigación crítica y con el video del profesor José Darío Herrera (2012) sobre la sistematización ${ }^{2}$.

En el caso de Torres, el hecho de que los estudiantes en su mayoría no lo hubieran leído, condujo a que se hiciera en el espacio del encuentro. Esta circunstancia fue importante, pues esa lectura resultó ser una muy buena estrategia que generó debate y participación y los acercó a estas otras posibles formas de investigación en nuestro campo.

Además, como estrategia para la lectura, implementé pruebas escritas, que al final, sin otras estrategias, no garantizan nada. Con esto, cerramos esta parte del seminario. Fue importante el diseño de unos ensayos sobre la pertinencia de nuestro campo. Creo que representó una acción acertada, pues permitió dejar registro de lo que plantean los estudiantes, sobre lo que considero, la pertinencia social de la educación física, la recreación, el deporte y la actividad física en contextos de paz y posconflicto.

Un segundo momento del seminario lo centramos específicamente en el desarrollo de la ruta de la sistematización. Fue una estrategia central adelantar todo el recorrido desde la recuperación de una práctica real de cada estudiante. Sobre este presupuesto, iniciaron con la definición de los objetos, los objetivos, los ejes, la línea del tiempo y la información existente de las experiencias. En esta parte se evidencia la problematización de los estudiantes enfrentados al tema de la participación de las personas con las que trabajaron en sus proyectos. Quizás por las formas predominantes, más centradas en las capacidades físicas y desde metodologías preferentemente directivas, en que al final es el profesor el que mide los avances a partir de indicadores tales como aumento de fuerza, el consumo de oxígeno máximo y el mejoramiento de las capacidades físicas. Los alumnos desarrollaron la planeación del proceso, frente a la identificación de los registros que tenían y los que hacían falta acopiar. Debido a que muchos de ellos ya no están en esos lugares, la posibilidad de programar encuentros, entrevistas, conversatorios, etc., en ocasiones era demorada, lo que hizo que la dinámica del seminario se diversificará y los procesos se centraran más en el acompañamiento.

2 ¿Qué es la sistematización de experiencias? Disponible en https://www.youtube.com/watch?v=9w1mg3Q7RFw. 
Un aspecto que vale la pena mencionar, es la situación de un pequeño grupo de estudiantes que no llegan a los últimos encuentros, debido a que consiguen trabajos en la organización de eventos deportivos y recreativos o son contratados en lugares para la temporada Navideña. Esto hace, que el acompañamiento, en algunos casos, se haga vía telefónica o por e-mail.

El final del proceso fue la entrega de los trabajos y la evaluación del seminario por parte de los estudiantes, buscando identificar desde su perspectiva, el aporte a su formación profesional y la pertinencia de este tipo de investigación para el campo.

Un aspecto adicional que cabe referenciar, es que el seminario se trabajó los días lunes, pues en Colombia, casi la totalidad de los festivos se pasaron para este día. Esto, asociado al hecho de que solo se cuente con 32 horas distribuidas en 16 encuentros de dos horas cada uno, hace que como en el caso del trabajado en el segundo semestre del 2016, prácticamente se pueda trabajar un $60 \%$ de lo inicialmente programado.

\section{Incidencia de las formas de la investigación en el programa educación física, recreación, deporte y actividad física de la Universidad de Antioquia}

Quizás uno de los aspectos emergentes más significativos del análisis de la información acopiada para la realización de esta sistematización, fue que casi en su totalidad los estudiantes manifestaran que era la primera vez que se acercaban a este tipo de investigación. En el semestre VII, después de haber trasegado por rutas metodológicas cualitativas y cuantitativas. Esto es muy significativo para la reformulación del seminario, que solo cuenta con 32 horas que terminaron siendo 24. Es decir, que además de la barrera del tiempo, los alumnos Ilegan al seminario sin ningún acercamiento a la perspectiva. Al respecto algunos de sus comentarios son ilustrativos:

\footnotetext{
“Llegué con muy poca información del tema a tratar, con una idea que salió de una conversación que tuve con un compañero de énfasis, el cual estaba realizando su tesis de grado por medio de una sistematización" (MR, est., 3).

"En cuanto a la sistematización de experiencias no tenía la idea como se trabajaba dicha manera de investigar" (MR, est., 6).

"Expectativas frente a un nuevo lineamiento de la investigación, conocimiento previo sobre la investigación, muy poca información sobre la sistematización de experiencias" (MR, Est., 13).
}

Esta situación configura un bosquejo de las formas predominantes, que se corresponden con las principales tendencias como la salud y el deporte, mayoritariamente centradas en modalidades empíricas e interpretativas de investigación, en que evidentemente perspectivas como la sistematización de experiencias tienen poco reconocimiento. La importancia de su evidenciación, es mostrar su incidencia en el proceso del seminario VII. En otras palabras, las rutas metodológicas para la producción de conocimiento con mayor registro portan concepciones de sociedad, de ser humano y del objeto mismo del 
campo. Lo que evidencia la necesidad de fortalecer el primer momento de su desarrollo, en relación con el tratamiento del tema, que en este contexto, pareciera no requerir ninguna discusión. O sea, es algo dado que no requiere reflexión ni problematización. Esto ha conducido a una mayoritaria presencia de procesos centrados en las técnicas en detrimento de propuestas orientadas a lo ontológico y lo epistemológico. Es decir, la identificación de la ubicación de lo metodológico en líneas que inciden en la comprensión de los contextos. Esto se hace explícito en comentarios de los estudiantes:

“Con base en los diferentes tipos de investigación. Un conocimiento elemental de construcción de proyectos de investigación en lo teórico. Pero si hay un gran vacío teórico" (MR, est., 1).

Llego con muchas ideas sobre el cómo investigar, algunas de ellas muy bien establecidas y otras un poco flojas y no muy claras. Llego con muchos vacíos y un poco de apatía por los procesos investigativos, un poco quizá por las malas experiencias en anteriores seminarios pero con una alta expectativa por el nuevo conocimiento. (MR, est., 8).

Se llega al seminario con un acumulado de cinco semestres de formación investigativa de tipo cualitativa, sin embargo no es muy significativa debido a que los procesos desarrollados en estos cursos nunca llegaron a procesos de análisis y mucho menos a una presentación final. Anteriormente los cinco cursos anteriores no tienen una concordancia que permiten desarrollar un verdadero proceso investigativo (MR, Est., 15).

Con lo anterior evidenció una situación que es oportuno tratar. El horizonte de formación, que articulado con la universidad investigadora, busca la detección y preparación de investigadores que lleguen a los grupos adscritos a la dependencia. Es decir, la apuesta de formar para la investigación, más que la investigación formativa, que apunta a la potenciación de la capacidad de indagación de los profesionales en los escenarios de actuación. Incluso podría plantearse que esta última además contribuiría a la formación de estudiantes para la práctica investigativa. Esto da entrada a la pregunta por el interés investigativo en el currículo. Es necesario formar también a los aprendices para problematizarse frente sus propias prácticas, las que realiza cotidianamente y que sin reflexión, pueden caer en la instrumentalización y posiblemente en la repetición acrítica.

De acuerdo con Mejía:

A este tipo de personas, desde distintas reflexiones se le ha denominado "profesionales prácticos en la acción" y con ello se intenta decir que son personas cuyo quehacer práctico, así tenga una fundamentación detrás de lo que hace, la preocupación principal no es la reflexión y el conocimiento. Pero además, el caso en el que se desarrollan proyectos específicos o se tiene un nivel de intervención sobre grupos humanos concretos, nos encontramos que pareciera que lo que hacen es repetir un libreto pre-diseñado y como tal vendrán a ser una especie de técnicos de procesos diseñados por otros y que ellos se convertirían en solo ejecutores (2013, p. 17). 
En este punto considero que la sistematización cumple un papel fundamental porque lo central de nuestra profesión son las prácticas en donde es normal la presencia de los Ilamados "empíricos": exdeportistas, practicantes de las diversas modalidades de la actividad físicas y otros campos que se desempeñan en ámbitos específicos de nuestro quehacer. Es decir, la posibilidad de una práctica problematizada y reflexionada relacionada explícitamente con horizontes de bienestar y buen vivir.

\section{Procesos didácticos en la Formación investigativa-investigación de profesionales}

El seminario de investigación VII se inscribe en la opción de la investigación formativa, que de acuerdo con Restrepo (2008) corresponde a la perspectiva que hace uso de la investigación en la docencia universitaria para acercarla a los estudiantes y formarlos en ella. En esta misma dirección Sánchez (2014) pone su atención en las formas de la enseñanza para la formación en investigación. Según sus planteamientos, estas deben plantearse desde lo teórico-práctico, como horizonte en la construcción de estrategias para la enseñanza de la investigación en las universidades. De acuerdo con Restrepo (2003) y Sánchez (2014), los procesos de formación de investigadores se dan a partir de dos tendencias principales: la formación para la investigación y la investigación formativa.

Restrepo plantea la formación para la investigación de la siguiente manera:

Hablar de investigación formativa es hablar de formación investigativa o del uso de la investigación para formar para investigar, pero en la investigación o a través de la investigación, con miras a aprender a investigar investigando, aunque esta actividad no conduzca necesariamente a descubrimiento de conocimiento nuevo y universal. Toda investigación formativa es formación en investigación, pero no toda formación en investigación es investigación formativa. El propósito de ésta última es más pedagógico que productivo de conocimiento nuevo (2008, p.3).

En la segunda tendencia plantea Sánchez que en la actualidad los procesos de formación para la investigación, corresponden mayoritariamente a un tipo de enseñanza formal, en que predomina un interés conceptual y documental. Ante esta situación, Ilama la atención sobre la perentoriedad de activar la promoción de formas otras de aprendizaje inspiradas en un modelo práctico y crítico de la didáctica de la investigación (2014).

En este contexto, en la recuperación de la experiencia, se evidenció que en lo didáctico el seminario VII se inscribe en la propuesta de la investigación formativa, de aprender a investigar investigando. Y más allá, a través de la recuperación crítica de una práctica propia.

A continuación presento aspectos emergentes en términos de lo didácticometodológico en el desarrollo del seminario 


\section{Potencialidad de la sistematización de experiencias como estrategia para aprenderla}

La decisión de proponer la ruta misma de la sistematización de experiencias como forma de acercarse a ella, es coherente con la perspectiva de la investigación formativa. Si a investigar se aprende investigando, el recorrido metodológico de la propuesta para el seminario fue pertinente, en el escenario de la formación de profesionales en el campo específico. En esta dirección, este tipo de investigación en sus elementos constitutivos permite problematizar la concepción de pesquisa. Es decir, acercase a referentes en que se reconoce el valor de las prácticas, la importancia de sus actores, la reivindicación de lo local y sobre todo el trabajo desde sus propios procesos. Un ejemplo de esto se evidencia en lo que dijo uno de los estudiantes:

"Se realiza ejercicios de reflexión donde se modifica ese paradigma para entender que la investigación va más lejos de un resultado" (MR, est., 10).

Otro comentario que muestra esta situación es:

Como todos los seminarios trabajamos a partir del tipo de investigación, pero en lo particular la sistematización es un método gratificante, por el hecho de trabajar con una experiencia propia. Aprendí mucho de este método por la forma en que se planteó, se presta para la interiorización de los temas vistos (Ev, est., 13).

Esto contribuye a que los encuentros y asesorías sean sobre situaciones reales, en que los estudiantes han participado. Trabajar a partir de experiencias propias los motiva y permite que los espacios de reunión sean más participativos y dinámicos. Esto lo manifiestan como uno de los factores favorecedores. En términos de la formación para la investigación, es uno de los principales rasgos de la sistematización. Principalmente para nuestros profesionales, predominantemente prácticos.

Otro asunto que se hace visible de la estrategia es la posibilidad que se genera en la perspectiva epistemológica de la sistematización, para problematizar los referentes con los Ilegan los estudiantes. Con formación en perspectivas de la investigación tanto positivistas como interpretativas que muchas veces no problematizan los contextos, encontrarse con una óptica que permite ubicar en el espacio complejo, las prácticas, los cuestiona y amplía la mirada a otros ámbitos que generalmente no son tenidos en cuenta. El comentario de uno de los estudiantes evidencia tal situación:

"A lo largo del curso, con las lecturas y con lo expuesto con el profesor se abrieron las puertas para pensar en realizar una investigación que no esté tan amarrada a las lógicas investigativas de las ciencias naturales" (MR, est., 19).

También la sistematización de experiencias en los procesos de formación para la investigación, posibilita visibilizar lo local y situado. Se hace presente la tensión entre 
concepciones del conocimiento frente a lo espacial. La ruta permite ir poniendo de presente estos temas que en últimas son regularidades no solo de esta forma de indagación sino de la epistemología misma. Así los estudiantes se confrontan con otras formas propias de la investigación en los procesos de formación de profesionales. Las predominantes en los proyectos finales de grado y en los niveles de especialización y maestría.

Un tercer elemento que recojo de la experiencia es haber orientado el seminario desde la ruta de la sistematización: su desarrollo corresponde a su paso a paso. Fundamentalmente en el segundo momento, cuando los estudiantes ya han elegido su práctica a sistematizar y comienza la reconstrucción, la identificación de los registros con los que cuentan y la información que pudiera faltar. Este es el punto en el que vuelven al campo a trabajar con los grupos con los que interactuaron. Definen estrategias para compartir con ellos y diseñan formatos. En muchos casos esta labor se dificulta por no estar ya trabajando en esos lugares.

Es pertinente visibilizar las dificultades de algunos alumnos para el diseño de sus estrategias de recolección de la información. Situación que Ilama la atención por la trayectoria que los antecede en su formación en investigación e implica la necesidad de reforzarla en futuros seminarios. También se resalta en este momento el hecho de que unos pocos estudiantes recurran a algunas maneras de lo deductivo para sus análisis. Por ejemplo, que empiecen el trabajo con un marco referencial y propongan muestras o variables. Tema que requerirá de una mayor cuidado en procesos didácticos venideros.

El último momento fue el de la construcción del informe donde todos escriben sus documentos. Considero que en esta última etapa emerge una de las grandes dificultades de estudiantes en formación, pero también de los profesionales del campo en general y es el problema de la escritura. Por lo que se ve en muchos de los textos, existe una limitación en los procesos escriturales que conduce a que no se logre plasmar su riqueza e importancia.

En general la estrategia de aproximarse a la sistematización de experiencias a través del recorrido por ella misma, representa para el caso específico de la formación de profesionales en educación física de la Universidad de Antioquia, una posibilidad de acercarse a otras perspectivas tradicionalmente poco reconocidas. Esta forma de indagación porta elementos constitutivos de formas otras para la producción de conocimiento. Es decir, el paso por las temáticas está lleno de aspectos que ponen en tela de juicio la hegemonía de modos en que se desconocen los contextos, se ve a las personas como objetos y se declara la neutralidad de la investigación y los investigadores. En este sentido la sistematización de experiencias representa un tipo de pesquisa crítica, que entra a jugar en la tensión con otras ópticas en el cometido de formar profesionales con capacidad de incidir en los entornos donde desarrolla sus prácticas.

En conclusión, la ruta de la sistematización de experiencias representa una propuesta didáctica metodológica potente y pertinente para los procesos de investigación formativa en el pregrado de educación física de la Universidad de Antioquia, más allá de la formación para la investigación a través de la aplicación de métodos y técnicas. El currículo del programa de educación física debería apostar por procesos de investigación formativa. De esta manera contribuiría tanto a la formación de investigadores para los 
grupos de la dependencia, como de profesionales que estén en capacidad de reflexionar permanentemente su quehacer, para mejorarlo cada día en beneficio propio y en el de los grupos humanos con los que trabajan. Más allá de esto, considero que la sistematización de experiencias es una alternativa de la mayor pertinencia para trabajar nuestro campo, sobre todo desde perspectivas integrales que posibiliten trascender las miradas instrumentales presentes y posicionadas en los procesos de formación.

Para finalizar, quiero hacer mención de un aspecto que no solo apareció en el segundo semestre de 2016 sino en otros seminarios anteriores. La referencia hecha por los alumnos sobre que este seminario debería estar en los primeros semestres, dado que al igual que la investigación, la práctica está presente en un alto porcentaje del proceso de formación. Al parecer algunos estudiantes identifican la afinidad entre la sistematización de experiencias y las prácticas.

Al respecto las siguientes menciones al tema por parte de algunos estudiantes:

“Pienso que el eje es muy importante, se debería ver antes de ir a las prácticas, nos da un nuevo recrear de nuestra profesión" Ev, est., 11).

"Proceso que aporta enormemente a la formación docente. En mi opinión este seminario debería ser visto cuando iniciamos prácticas, pues es el método más acertado para una reconstrucción de nuestras experiencias" (Ev, est., 16).

Considero que de todas maneras, en medio de la poca presencia de perspectivas críticas en el proceso de formación profesional de la dependencia, es necesario emprender acciones que posibiliten un contexto de mayor equilibrio entre las diferentes ópticas de la investigación. La sistematización de experiencias representa una propuesta pertinente en la perspectiva de la investigación formativa, al contener en ella misma una serie de regularidades que problematizan aquellas formas instrumentales de la pesquisa más centradas en el aprendizaje de métodos

Una mención final, ilustra esta situación:

“Con bases en los diferentes tipos de investigación. Un conocimiento elemental de construcción de proyectos de investigación en lo teórico. Pero si hay un gran vacío teórico" (MR, est., 1).

\section{Aprendizajes, conclusiones y recomendaciones}

El proceso de sistematización de la experiencia del seminario VII de investigación, realizado en el segundo semestre de 2016, fue para mí de la mayor valía. El recorrido hecho mediante el análisis de los diferentes momentos, de la mano de registros (rastros como el documento del seminario, las matrices de reconstrucción, evaluación y factores favorecedores y obstaculizadores), me permitió profundizar en el proceso vivido, visibilizar aspectos que emergían como elementos constitutivos, ampliar la comprensión de lo realizado, aprender más sobre la sistematización de experiencias, 
la didáctica de la investigación y las lógicas de la producción de conocimiento. Un proceso realmente emocionante, en que fue posible descubrir cómo emergían cosas que aunque se consideran implícitas, no eran tan evidentes. Ver cómo algo planteado para un seminario de investigación, en una ciudad y en un programa concreto de formación de profesionales en educación física, permitía no solo profundizar en sus lógicas propias, sino observar cómo me daban elementos para el análisis de los enfoques del campo y sus correspondientes tendencias frente a la producción del conocimiento. En esta dirección este proceso de sistematización de experiencias contribuye al fortalecimiento del seminario. La identificación de sus principales elementos constitutivos posibilita una acción intencional hacia su robusteciendo, pues al entender los momentos y la relación entre ellos puedo imaginar nuevas formas metodológicas que contribuyan a generar espacios más adecuados. El recorrido por el seminario permitió igualmente reconocer su valioso papel, más allá del tema de la sistematización, de la didáctica de la investigación en un currículo que cuenta con ocho seminarios. Es decir, que sus regularidades (las prácticas, lo situado, lo participativo, etc.), son aspectos centrales de una didáctica que se muestra como irrupción en el panorama de las formas predominantes en la formación de los y las profesionales del programa de educación física de la Universidad de Antioquia. Pero además, comprender que somos resultado de lo que pasa en el país y en gran parte de América Latina sobre de la situación de los procesos de investigación.

Considero que la didáctica de la investigación será en adelante un referente desde el cual potenciar procesos que contribuyan a la discusión sobre este componente en el Instituto Universitario de Educación Física en la Universidad de Antioquia. Esta sistematización aportó al fortalecimiento y reformulación de temáticas y posibilidades metodológicas para que los estudiantes encuentren un mejor escenario para su formación profesional y amplíen sus miradas frente al tema de la investigación. Una ruta que permita mostrar los contextos diversos y abigarrados de nuestras realidades.

Otro aspecto a resaltar, fue el de proponer el contexto del posconflicto y la construcción de la paz como elemento central, que al final permitió amplificar la discusión sobre el objeto del campo, pero que al final se diluyó y fue poco representativo en los procesos de sistematización. Situación que considero propia de la radicalización de la coyuntura, de los imaginarios construidos por diversas posturas ideológicas y la postura de muchos de nuestros estudiantes sobre la incompatibilidad entre el campo y la política

En referencia a las conclusiones, es valioso mencionar la significativa contribución de la mirada al seminario desde el eje de la didáctica, pensado en principio como posibilidad para el fortalecimiento del seminario VII, pero que en realidad me puso frente a la didáctica de la investigación. Esto me permitió ampliar la óptica frente a los diferentes temas tratados en el análisis y considerar esta forma de pesquisa como clave para la ampliación del horizonte de la formación del profesional. También hizo posible la emergencia de esta manera de indagación, más allá de la apropiación de sus regularidades, de identificar su importancia y pertinencia para formar investigadores. Hoy tengo una más amplia comprensión sobre la sistematización de experiencias y su potencial formativo para la investigación, lo que lleva a pensar en nuevas posibilidades. 
Como recomendación, iniciar procesos en torno a la investigación en la dependencia. De acuerdo con lo encontrado, se hace necesario invitar a profesores y profesoras al frente de los diferentes seminarios, para buscar algunos acuerdos que permitan aprovechar la posibilidad de sus ocho semestres en el proceso de formación. Para el caso del Seminario VII, mejorar sustancialmente los registros, de tal manera que un nuevo ejercicio de este tipo pueda contar con un mayor soporte, pero también para fortalecer los procesos de formación en investigación.

\section{REFERÊNCIAS}

CRISORIO, Ricardo. Educación Física e identidad: conocimiento, saber y verdad. VIREF, Universidad de Antioquia. Disponible en: http://viref.udea.edu.co/contenido/publicaciones/ memorias_expo/educacion_fisica/ef_identidad.pdf. Acceso en 30 jun. 2017.

JARA, Oscar. Para sistematizar experiencias: una propuesta teórica y práctica. San José de Costa Rica: Centro de estudios y publicaciones, Alforja, 1994

Mejía, Marco. La sistematización. Empodera y produce saber y conocimiento. BogotaColombia: Ediciones desde abajo, 2013

PINILLOS, Jesús. La constitución del campo de la educación física en Colombia en el periodo comprendido entre 1968 y 1991. Recorde: Revista de História do Esporte, v. 4, n. 2, p. 1-23. 2011.

SÁNCHEZ, Ricardo. Enseñar a investigar. Una didáctica nueva de la investigación en ciencias sociales y humanas. Ciudad de México: Universidad Nacional Autónoma de México. Instituto de Investigaciones sobre la Universidad y la Educación, 2014.

TABARES, Fernando, MOLINA, Víctor; CUERVO, Iver. Guía para la sistematización de experiencias. Recuperación del saber y del ser en ocio, deporte, educación física y actividad física. Disponível em http://www.cepalforja.org/sistem/bvirtual/?p=1159. Acceso en: 25 jun. 2017.

TORRES, Alfonso. Producción de conocimiento desde la investigación crítica. Nómadas. Colombia, Bogotá, n. 40, p. 68-83, 2014

VAZ, Alexandre. Aprender a produzir e mediar conhecimentos: um olhar sobre a pratica de ensino de Educacão Fisica. Motrivivencia, v. 21, n. 13, p. 11-34, 1999

\section{Fuentes primarias}

Matriz de reconstrucción del Seminario: MR

Matriz Evaluación Seminario: Ev.

Matriz identificación factores favorecedores y obstaculizadores de la experiencia. MFFO.

Recebido em: Julho/2017

Aprovado em: Outubro/2017 